\title{
A 385-500 GHz Sideband-Separating (2SB) SIS Mixer Based on a Waveguide Split-Block Coupler
}

\author{
Mamoru Kamikura • Yu Tomimura • Yutaro Sekimoto • \\ Shin'ichiro Asayama • Wenlei Shan • Naohisa Satou • \\ Yoshizou Iizuka • Tetsuya Ito • Toshiaki Kamba • \\ Yasutaka Serizawa • Takashi Noguchi
}

Published online: 18 September 2007

(C) Springer Science + Business Media, LLC 2007

\section{Erratum to: Int J Infrared Milli Waves \\ DOI 10.1007/s10762-006-9052-4}

In the original article the following figures were inadvertently printed as low resolution and therefore are unreadable. They are reprinted on the following pages along with the captions.

The online version of the original article can be found at: http://dx.doi.org/10.1007/s10762-006-9052-4.

M. Kamikura $(\bowtie) \cdot$ Y. Tomimura $\cdot$ Y. Sekimoto $\cdot$ Y. Serizawa

Department of Astronomy, School of Science, The University of Tokyo, 2-21-1 Osawa, Mitaka, Tokyo 181-8588, Japan

e-mail: kamikura.mamoru@nao.ac.jp

M. Kamikura $\cdot$ Y. Tomimura $\cdot$ Y. Sekimoto $\cdot$ S. Asayama $\cdot$ W. Shan $\cdot$ N. Satou $\cdot$ Y. Iizuka $\cdot$ T. Ito $\cdot$

T. Kamba $\cdot$ Y. Serizawa $\cdot$ T. Noguchi

Advanced Technology Center and ALMA-J project office, National Astronomical Observatory of Japan,

National Institutes of Natural Sciences, 2-21-1 Osawa, Mitaka, Tokyo 181-8588, Japan

W. Shan

Purple Mountain Observatory, Chinese Academy of Sciences, 2 West Beijing Road, Nanjing 210008, China 


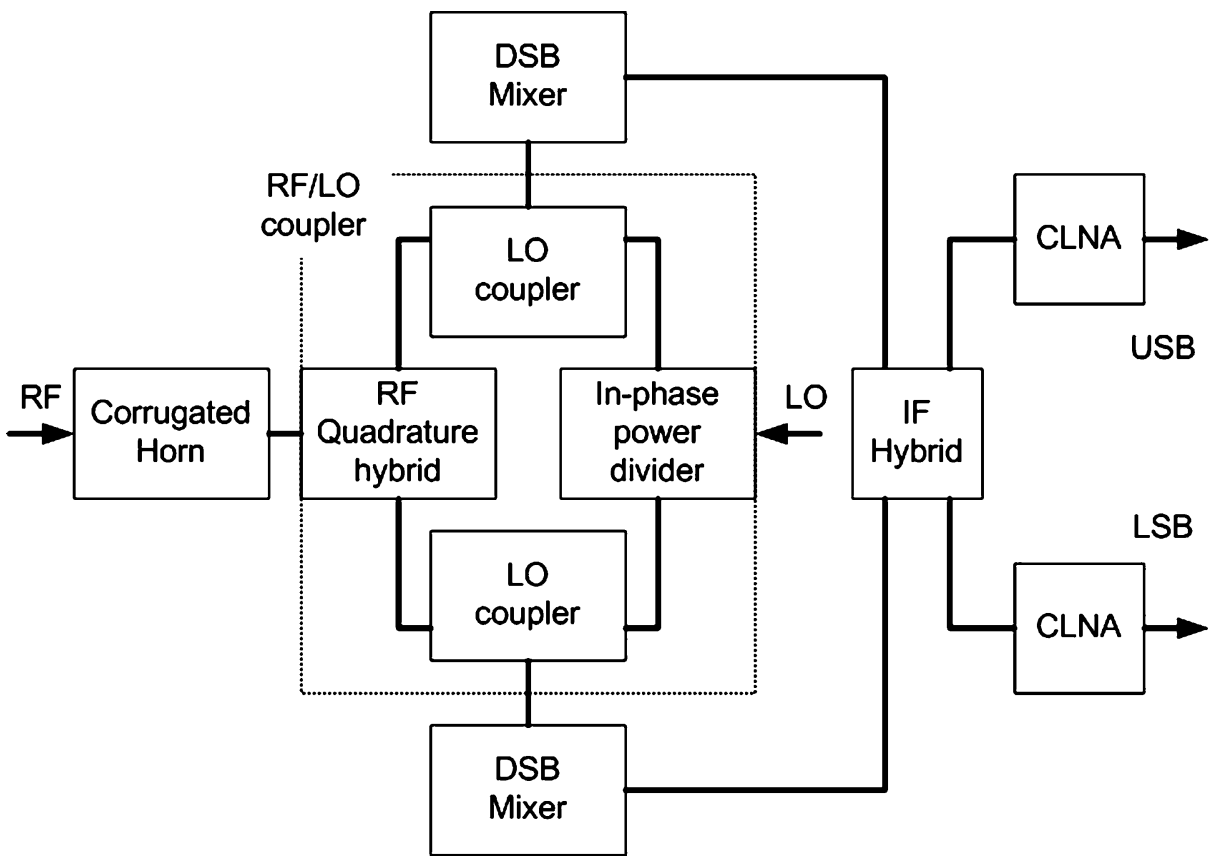

Fig. 1 Block diagram of a 2SB mixer.

Fig. 2 Diagram of the RF quadrature hybrid. Dimensions are in $\mu \mathrm{m}$.
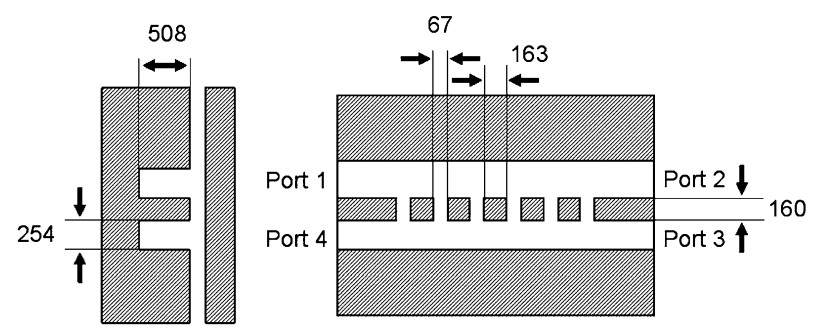
a

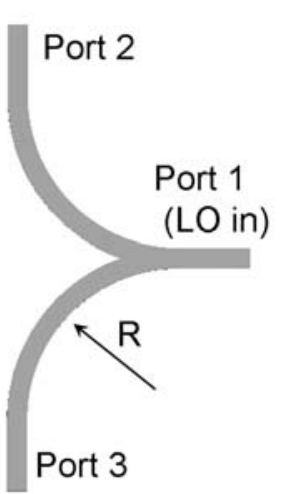

b

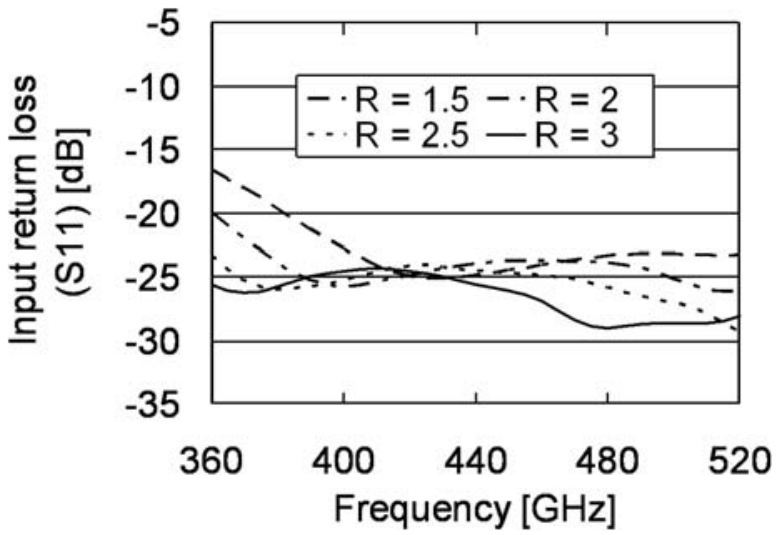

Fig. 4 (a) Diagram of the in-phase power divider. This is an E-plane Y-junction. The waveguide size is $508 \mu \mathrm{m} \times 254 \mu \mathrm{m}$. (b) Simulated results of the input return loss of the in-phase power divider when the radius is varied from $1.5 \mathrm{~mm}$ to $3.0 \mathrm{~mm}$.

Fig. 6 Diagram of the LO coupler. Dimensions are in $\mu \mathrm{m}$.
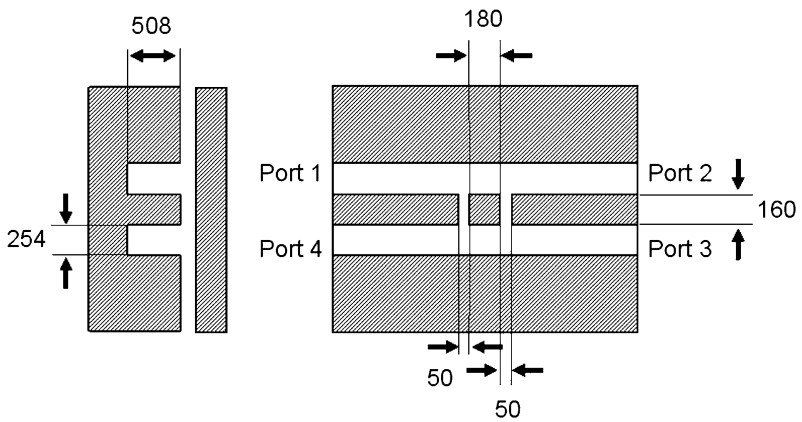

Fig. 8 Photograph of the RF/LO coupler.

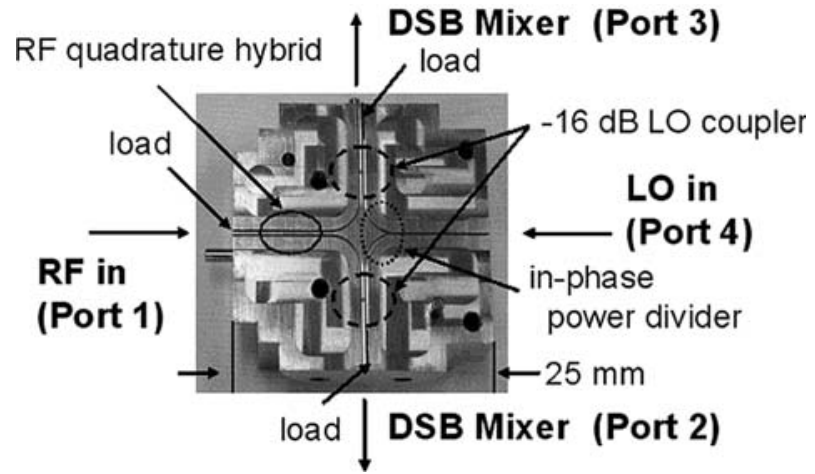


Fig. 9 Photograph of the RF quadrature hybrid. The RF quadrature hybrid is a 6 branch-line coupler. The dimensions are in $\mu \mathrm{m}$. The values with and without the parentheses show the design and optically measured values, respectively.
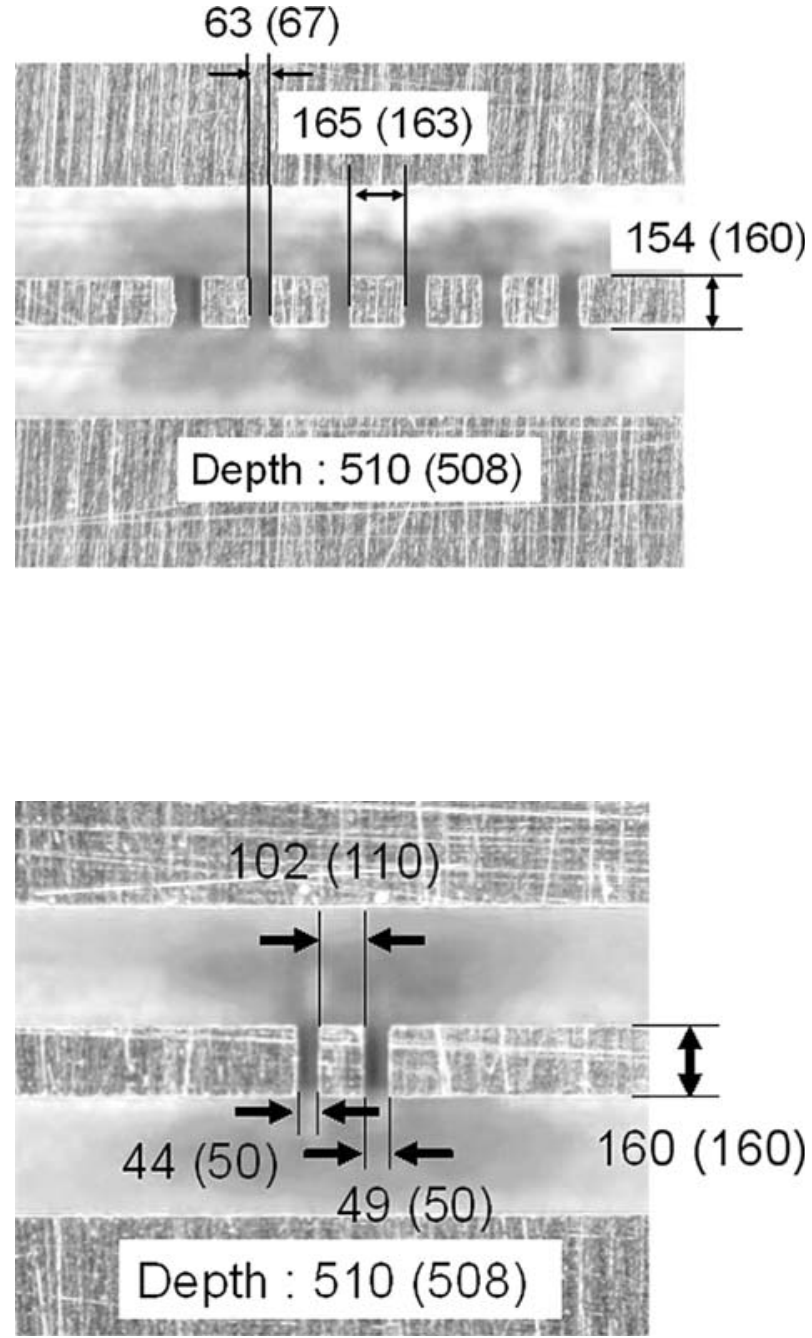